University of Wollongong

Research Online

Faculty of Engineering - Papers (Archive)

Faculty of Engineering and Information

Sciences

2006

\title{
Magneto-optical imaging of the magnetization process in colossal magnetoresisitive lanthanum manganite
}

\author{
A. Polyanskii \\ University of Wisconsin, Madison, USA \\ Xiaolin Wang \\ University of Wollongong, xiaolin@uow.edu.au \\ Qiwen Yao \\ University of Wollongong, qy75@uow.edu.au \\ S. X. Dou \\ University of Wollongong, shi@uow.edu.au \\ Z. W. Lin \\ University of Technology, Sydney
}

See next page for additional authors

Follow this and additional works at: https://ro.uow.edu.au/engpapers

Part of the Engineering Commons

https://ro.uow.edu.au/engpapers/157

\section{Recommended Citation}

Polyanskii, A.; Wang, Xiaolin; Yao, Qiwen; Dou, S. X.; Lin, Z. W.; and Zhu, J. G.: Magneto-optical imaging of the magnetization process in colossal magnetoresisitive lanthanum manganite 2006.

https://ro.uow.edu.au/engpapers/157 
Authors

A. Polyanskii, Xiaolin Wang, Qiwen Yao, S. X. Dou, Z. W. Lin, and J. G. Zhu 


\title{
Magneto-optical imaging of the magnetization process in colossal magnetoresisitive lanthanum manganite
}

\author{
A. Polyanskii \\ Applied Superconductivity Centre, University of Wisconsin, Madison, Wisconsin 53706 \\ X. L. Wang ${ }^{\text {a) }}$ \\ Spintronic and Electronic Materials Group, Institute for Superconducting and Electronic Materials, \\ University of Wollongong, Northfields Avenue, New South Wales 2522, Australia \\ Q. W. Yao and S. X. Dou \\ Institute for Superconducting and Electronic Materials, University of Wollongong, Northfields Avenue, \\ New South Wales 2522, Australia \\ Z. W. Lin and J. G. Zhu \\ Faculty of Engineering, University of Technology, Sydney, NSW 2007, Australia
}

(Presented on 31 October 2005; published online 28 April 2006)

\begin{abstract}
To gain insight into the origin of the colossal reduction of resistance in response to magnetic field in colossal magnetoresistance manganite, the magnetic field induced transition in ferromagnetic $\mathrm{La}_{0.7} \mathrm{Ca}_{0.3} \mathrm{MnO}_{3}$ was studied using a high-resolution magneto-optical imaging (MOI) technique. The MO images were captured in various magnetic fields over a wide temperature range for both highly dense samples with strong-link grain boundaries and porous samples with weak-link boundaries. Formation and evolution of magnetic domains as a function of field or temperature were clearly observed around and far below the Curie temperature $T_{C}=240 \mathrm{~K}$. Ferromagnetic areas tend to grow to large sizes and finally join together at the expense of paramagnetic areas as the field increases or temperature decreases for strong-link samples. A sharp magnetoresistive transition is observed when the sample changes from a paramagnetic insulator to a metallic ferromagnetic phase in the vicinity of $T_{C}$. In contrast, the porous samples showed magnetoresistance over a wide temperature range and exhibited a remarkable grain boundary related magnetization process in addition to magnetization within grains. A close correlation is found between the magnetization process observed by MOI and magnetoresistance measurements. Our MOI results indicate that the strong-link or weak-link grain boundaries are responsible, respectively, for magnetoresistance occurring either only in the vicinity of the ferromagnetic transition or over a very wide temperature range. (C) 2006 American Institute of Physics. [DOI: 10.1063/1.2177393]
\end{abstract}

The rediscovery of colossal magnetoresistance (CMR) in manganite perovskite in conjunction with fascinating physical properties, such as charge and spin orderings, abundant magnetic phases, electronic phase separation, etc., has intrigued researchers and motivated extensive studies worldwide. The study of the CMR materials has become one of the mainstream areas in solid state physics, as happened previously with cuprate high temperature superconductors. ${ }^{1,2}$ One of the major challenges is to elucidate the origin of CMR. The most likely mechanism comes from the model of electronic phase separation (EPS). ${ }^{3,4}$ When changing from paramagnetic (PM) to ferromagnetic (FM) phases around the Curie temperature $T_{C}$, CMR materials undergo a nonhomogeneous magnetization process and simultaneously form ferromagnetic regions that consist of a nanoscale secondary electronic phase whose conductivity is much greater than that of the paramagnetic regions, leading to a drop in resistance. The resistance can be further decreased by either applying an external magnetic field or by reducing the temperature further, resulting in the actual so-called CMR effect.

\footnotetext{
a) Author to whom correspondence should be addressed; electronic mail: xiaolin@uow.edu.au
}

Conductive ferromagnetic regions expand at the expense of paramagnetic insulating regions. However, it should be pointed out that some nanoscale insulating regions are always present under very high magnetic fields and at very low temperatures, as has been observed using scanning tunneling microscopy $(\mathrm{STM}) .^{5}$ The EPS picture explains the magnetoresistance in the manganites in which CMR occurs only within a narrow temperature range in the vicinity of the transition from PM to FM and diminishes quickly with decreasing temperature. However, in most cases, the CMR is present over a very wide temperature range, from the Curie temperature down to very low temperatures. It has been argued that this is caused by the spin polarized tunneling effect ${ }^{6}$ through grain boundaries. It has been reported that strong-link and weak-link grain boundaries, respectively, seem to be responsible for CMR that appears only in the vicinity of $T_{C}$ and that which is present over a wide temperature range. ${ }^{7,8}$

In our previous work ${ }^{9}$ we have used the magneto-optical imaging (MOI) technique for direct visualization of the magnetization process in a highly dense $\mathrm{La}_{0.7} \mathrm{Ca}_{0.3} \mathrm{MnO}_{3}$ bulk sample showing CMR around $T_{C}=240 \mathrm{~K}$. MOI allows a direct observation of the formation of magnetic domains and provides some details of the local magnetization process, 
which is unattainable from global magnetization measurements. However, the special resolution of MOI in that study was not completely adequate due to the labyrinth stripe magnetic domain pattern in the indicator film with perpendicular magnetization.

In this work we present our studies using higher resolution MOI with an in-plane magnetized Bi-doped iron-garnet indicator ${ }^{10}$ for direct visualization of the magnetization process in a highly dense sample with strong-link grain boundaries and in a very porous sample with weak-link grain boundaries. The magneto-optical indicator utilizes Faraday rotation for the visualization of the normal component of the magnetic field on the on top surface of the sample to create a map of the magnetic induction at the sample surface.

Two different single-phase $\mathrm{La}_{0.7} \mathrm{Ca}_{0.3} \mathrm{MnO}_{3}$ (LCMO) samples were used for the present study: one, called the HD sample, was made by a partial melting process and is highly dense, while the other one is a very porous sample made using a conventional sintering process. The structural characteristics of both samples were studied in Ref. 8. The porous sample has grain sizes of about $100 \mu \mathrm{m}$ and wide weaklink grain boundaries. By contrast, the HD sample looks very dense and no clear boundaries can be seen.

The highly dense sample exhibited magnetoresistance (MR) only in the vicinity of $T_{C}$ at about $230 \mathrm{~K}$, while the porous sample revealed MR over a very wide range of temperatures from the $T_{C}$ down to $5 \mathrm{~K}{ }^{8}$ A very porous sample with very large grain boundaries (see Fig. 3) was selected for MOI observation. This enabled us to visualize the grain boundaries under an optical microscope.

Figure 1 shows the magneto-optical images of the normal component of the magnetic induction $B_{z}$ at the surface of the HD sample. The images from top to bottom on the left side were taken at temperatures of 250, 234, 228, and $220 \mathrm{~K}$, respectively, as the sample was cooled down from $300 \mathrm{~K}$ in a field of $60 \mathrm{mT}$. The images on the right side were taken in fields of 10, 20, 30, and $40 \mathrm{mT}$ at $234 \mathrm{~K}$. The bright areas in these images correspond to high values of $B_{z}$. It can be seen that in the presence of a constant field of $60 \mathrm{mT}$, the sample was paramagnetic at $250 \mathrm{~K}$, but as temperature decreased some local regions started to become ferromagnetic independently at $T=234 \mathrm{~K}$ (bright contrast). With further decreases in temperature, new ferromagnetic regions started to form. Those already formed regions expanded to merge with other regions and form large ferromagnetic regions at the expense of the paramagnetic regions. Finally, the magnetization process resulted in homogeneous magnetization throughout the whole sample at $T=220 \mathrm{~K}$. Further drops in temperature led to little change in the MO images. The observation of this process is in agreement with our previous work using low resolution MOI. ${ }^{9}$ It should be noted that these MO images indicate a close correlation with the electrical transport measurements where a significant drop in resistance takes place entirely within the 250-220 K temperature range.

At the fixed temperature of $234 \mathrm{~K}$ (see images on the right side of Fig. 1) where the resistance is at a maximum, the application of magnetic field favors the formation of ferromagnetic regions in a similar way to the effect of temperature discussed above. The brightness and areas of ferromag-
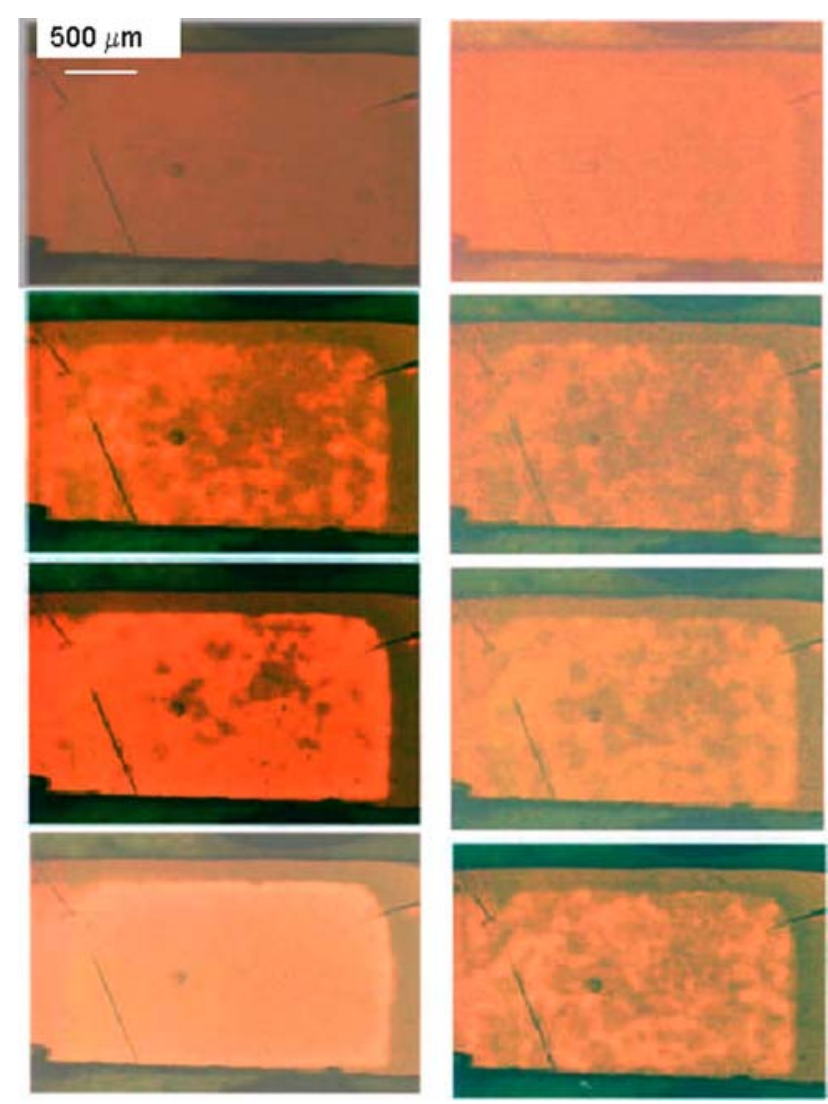

FIG. 1. (Color online) Magneto-optical images taken on the HD sample. Left column: images taken in a field of $60 \mathrm{mT}$ at temperatures of 250, 234, 228 , and $220 \mathrm{~K}$ (from top to bottom). Right column: images taken at $T$ $=234 \mathrm{~K}$ in fields of $10,20,30$, and $40 \mathrm{mT}$ (from top to bottom). The width of the samples is $2.77 \mathrm{~mm}$ (vertical direction).

netic regions were enhanced without any clear sign of merging at this temperature when the field increased from 10 up to $40 \mathrm{mT}$. The enhanced magnetization in the independent local areas closely corresponds to a significant drop in resistance under the application of field (for the HD sample), in agreement with what has been seen in a LCMO thin film under STM. ${ }^{7}$

In contrast, the very porous sample exhibited a remarkable grain boundary controlled magnetization process in the MO images shown in Fig. 2, in addition to the magnetization within grains. According to our observations, below $T_{C}$, individual grains separated by large grain boundaries gradually entered the ferromagnetic state as their brightness gradually increased with increasing magnetic fields. The regions located at grain boundaries remained weakly magnetic compared to the regions inside grains. This homogeneous magnetization only took place until the field increased to a certain value. This process occurred not only at the temperatures around $T_{C}$, but also always appeared over a very wide range of temperatures, from $T_{C}$ down to $4 \mathrm{~K}$. This is the most significant difference in the MO images between the very porous sample with large grain boundaries and the highly dense sample with invisible and strong-link grain boundaries.

A typical series of the MO images taken at $11 \mathrm{~K}$ for the porous sample in various fields are shown in Fig. 2. It can be 


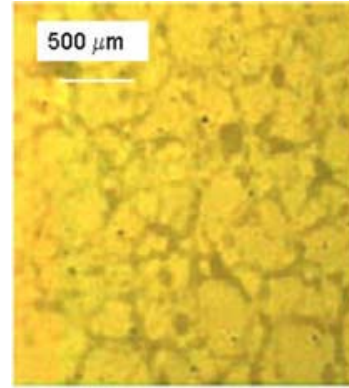

$B=40 \mathrm{mT}$

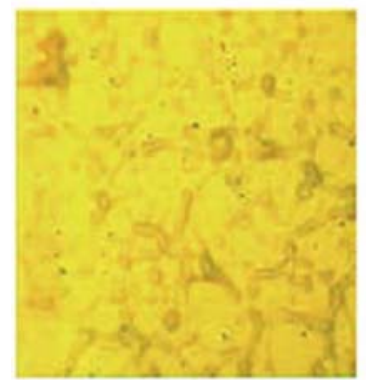

$\mathrm{B}=92 \mathrm{miT}$

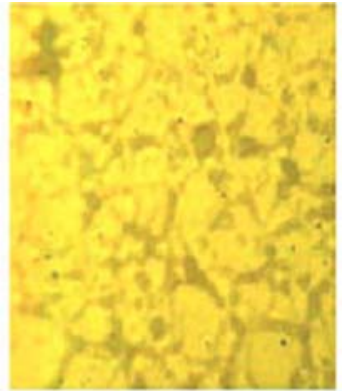

$\mathrm{B}=60 \mathrm{mT}$

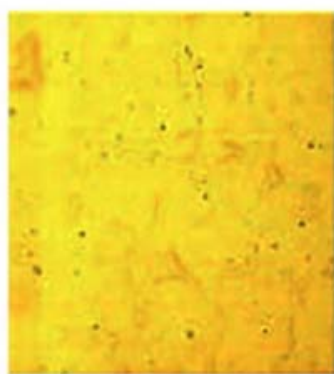

$B=144 \mathrm{mT}$
FIG. 2. (Color online) Magnetization process in porous sample at $11 \mathrm{~K}$. The dark contrast is located at grain boundaries.

seen that the regions at grain boundaries are darker than inside the grains in low fields of 40 and $60 \mathrm{mT}$, but eventually merge together with all other grains and boundaries in high fields. Such an evolution of the grain boundary associated magnetization process is closely correlated with the changes in resistance, which always exhibits a drop in magnetic field over a wide range of temperatures from $T_{C}$ down to $5 \mathrm{~K}$. The close relationship between the magnetization process and the magnetoresistance clearly indicates that weak-link grain boundaries are indeed responsible for wide-temperaturerange magnetoresistance in LCMO compounds.

Figure 3 presents an optical image of structural defects on the surface of the porous sample together with the corresponding MO image taken on the same part of the surface and scanning electron microscopy (SEM) images of the grain structure. The correlation between grain structure and $\mathrm{MO}$ contrast is very clear. Wide grain boundaries (width about 10-30 $\mu \mathrm{m}$ ) create dark contrast on MO images. With increasing field the dark contrast splits and shows the fine structure of grain boundaries (MOI in Figs. 2 and 3). It seems that these grain boundaries are responsible for magnetoresistance that occurs over a wide temperature range.

In summary, we have found that when cooling down from the paramagnetic to the ferromagnetic state for the $\mathrm{La}_{0.7} \mathrm{Ca}_{0.3} \mathrm{MnO}_{3}$ samples with strong-link grain boundaries, inhomogeneous ferromagnetic regions start to form and tend to grow to large sizes with the generation of new ferromagnetic regions. They are finally joined together at the expense of paramagnetic areas as the field increases or the tempera-
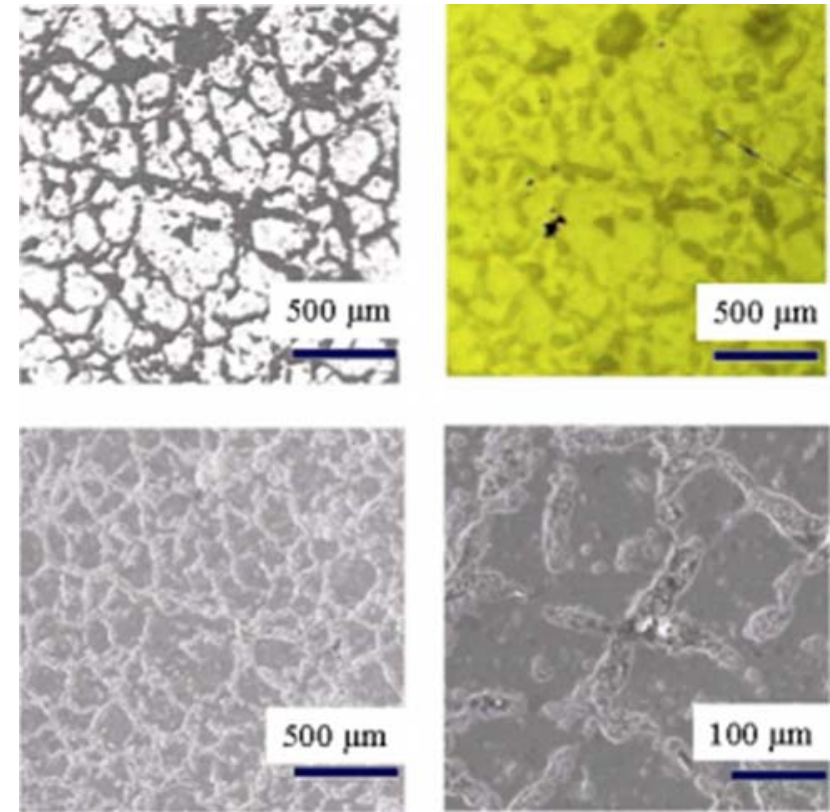

FIG. 3. (Color online) Grain structure on the surface of the porous sample and corresponding MO images. Top row: optical image (left) and MO image (right) taken under ZFC conditions at $T=200 \mathrm{~K}$ and $H=60 \mathrm{mT}$. Bottom row: SEM image of the grain structure (left) and wide grain boundaries (right).

ture further decreases. The magnetoresistance seen in these strong-link samples is caused by the reduction of the paramagnetic insulator phase and the increase of the metallic ferromagnetic phase around the Curie temperature. The magneto-optical images for the porous samples with weaklink grain boundaries exhibited a remarkable grain boundary controlled magnetization process. Strong-link or weak-link grain boundaries are responsible for magnetoresistance occurring either only in the vicinity of the ferromagnetic transition or over a very wide temperature range.

One of the authors (X.L.W.) thanks the Australian Research Council for funding support through Discovery projects DP0345012 and DP0558753 and is also grateful for the support from the Superconductivity Group at the University of Wisconsin.

${ }^{1}$ R. von Helm, R. Wecker, J. Holzapfel, B. Schultz, and K. Samwer, Phys. Rev. Lett. 71, 2331 (1993).

${ }^{2}$ J. M. D. Coey, Adv. Phys. 48, 167 (1999).

${ }^{3}$ A. Moreo, S. Yunoki, and E. Dagotto, Science 283, 2034 (1999).

${ }^{4}$ E. Dagotto, Nanoscale Phase Separation and Colossal Magnetoresistance, Springer Series on Solid-State Physics (Springer, New York, 2005).

${ }^{5}$ M. Fath et al., Science 285, 2034 (1999).

${ }^{6}$ H. Y. Huang, S.-W. Cheong, N. P. Ong, and B. Batlogg, Phys. Rev. Lett. 77, 2041 (1996).

${ }^{7}$ N. D. Matur et al., Nature (London) 387, 266 (1997).

${ }^{8}$ X. L. Wang et al., Appl. Phys. Lett. 73, 396 (1998).

${ }^{9}$ Z. W. Lin et al., Appl. Phys. Lett. 74, 3014 (1999).

${ }^{10}$ A. A. Polyanskii, D. M. Feldmann, and D. C. Larbalestier, in Handbook of Superconducting Materials, edited by D. Cardwell and D. Ginley (IOP, New York, 2003), Chap. C3.4, pp. 1551-1567. 